Meta

Journal des traducteurs

Translators' Journal

\title{
Le péritexte des traductions anglaises du Discours sur les Sciences et les Arts de Jean-Jacques Rousseau : la voix énarrative du traducteur
}

\section{Guy Rooryck et Lieve Jooken}

Volume 58, numéro 3, décembre 2013

URI : https://id.erudit.org/iderudit/1025053ar

DOI : https://doi.org/10.7202/1025053ar

Aller au sommaire du numéro

Éditeur(s)

Les Presses de l’Université de Montréal

ISSN

0026-0452 (imprimé)

1492-1421 (numérique)

Découvrir la revue

Citer cet article

Rooryck, G. \& Jooken, L. (2013). Le péritexte des traductions anglaises du Discours sur les Sciences et les Arts de Jean-Jacques Rousseau : la voix énarrative du traducteur. Meta, 58(3), 589-606.

https://doi.org/10.7202/1025053ar
Résumé de l'article

Des échanges et des transferts entre les nations européennes naît au xviii ${ }^{\mathrm{e}}$ siècle la conceptualisation d'un monde nouveau où la traduction joue un rôle médiateur indispensable. Recourant à l'appareil critique genettien, cette étude tente spécifiquement de définir une typologie des fonctions assumées par la voix du traducteur qui laisse une trace explicite ou implicite dans le discours traduit. Cette voix assume, dans l'appropriation du texte original, une fonction herméneutique définie ici comme " énarrative ». Le cas étudié est celui du Discours sur les Sciences et les Arts (1751), qui a propulsé Rousseau sur l'avant-scène de la République des Lettres et qui a connu pas moins de quatre traductions anglaises du vivant de l'auteur. Séduits par l'éloquence qu'il s'agit d'égaler et par la hardiesse du propos, traducteurs et commentateurs expriment tantôt leur perplexité, tantôt leur fascination. Les traductions de Bowyer (1751), Wynne (1752), d'un auteur anonyme (1760) et de Kenrick (1767) contiennent dans leur appareil péritextuel de précieuses indications sur la réception des idées qu'elles propagent. Les traducteurs font ainsi entendre leur voix énarrative tant dans l'appareil titulaire que dans des préfaces originales en anglais, assumant ainsi les fonctions méta-discursive, évaluative, argumentative et extra-diégétique que cet article contribue à définir.
Ce document est protégé par la loi sur le droit d'auteur. L'utilisation des services d’Érudit (y compris la reproduction) est assujettie à sa politique d'utilisation que vous pouvez consulter en ligne.

https://apropos.erudit.org/fr/usagers/politique-dutilisation/ 


\title{
Le péritexte des traductions anglaises du Discours sur les Sciences et les Arts de Jean-Jacques Rousseau: la voix énarrative du traducteur
}

\author{
GUY ROORYCK \\ Universiteit Gent, Gand, Belgique \\ guy.rooryck@ugent.be \\ LIEVE JOOKEN \\ Universiteit Gent, Gand, Belgique \\ lieve.jooken@ugent.be
}

\begin{abstract}
RÉSUMÉ
Des échanges et des transferts entre les nations européennes naît au XVIII ${ }^{\mathrm{e}}$ siècle la conceptualisation d'un monde nouveau où la traduction joue un rôle médiateur indispensable. Recourant à l'appareil critique genettien, cette étude tente spécifiquement de définir une typologie des fonctions assumées par la voix du traducteur qui laisse une trace explicite ou implicite dans le discours traduit. Cette voix assume, dans l'appropriation du texte original, une fonction herméneutique définie ici comme «énarrative». Le cas étudié est celui du Discours sur les Sciences et les Arts (1751), qui a propulsé Rousseau sur l'avant-scène de la République des Lettres et qui a connu pas moins de quatre traductions anglaises du vivant de l'auteur. Séduits par l'éloquence qu'il s'agit d'égaler et par la hardiesse du propos, traducteurs et commentateurs expriment tantôt leur perplexité, tantôt leur fascination. Les traductions de Bowyer (1751), Wynne (1752), d'un auteur anonyme (1760) et de Kenrick (1767) contiennent dans leur appareil péritextuel de précieuses indications sur la réception des idées qu'elles propagent. Les traducteurs font ainsi entendre leur voix énarrative tant dans l'appareil titulaire que dans des préfaces originales en anglais, assumant ainsi les fonctions méta-discursive, évaluative, argumentative et extra-diégétique que cet article contribue à définir.
\end{abstract}

\begin{abstract}
Building on a Genettian paratextual framework, this study explores a typology of the functions that define the Translator's voice in eighteenth-century British translations of philosophical discourse. In appropriating the original text, this voice assumes a number of hermeneutic functions that underpin what will be identified as the translator's "enarrative" mediation of the source text. The case analysed is the translation of Jean-Jacques Rousseau's Discours sur les Sciences et les Arts (1751), the work that launched the author's reputation in the Republic of Letters. Four English translations of the discourse - by Bowyer (1751), Wynne (1752), an anonymous author (1760) and Kenrick (1767) - were published in the course of the author's lifetime. The functions of enarrative voice will be traced in two peritextual instances, viz. the title page and the translator's preface, and will be categorized as meta-discursive, evaluative, argumentative and extra-diegetic. Trying to emulate the eloquence of the original, the translators oscillate between expressing their perplexity and their fascination towards its views, notably in their own prefaces, which qualify the univocality of the text the reader is about to read. Together the instances of Translator's voice provide a discursive record of the contemporary reception of Rousseau's ideas in Britain.
\end{abstract}

\section{MOTS-CLÉS/KEYWORDS}

Jean-Jacques Rousseau, voix du traducteur, énarration, péritexte, préface du traducteur Jean-Jacques Rousseau, translator's voice, enarratio, peritext, translator's preface 


\section{Traduction et péritexte: le Discours sur les Sciences et les Arts outre-Manche}

La traduction se laisse difficilement circonscrire et se prête d'autant plus facilement à la métaphore: passerelle, négociation, métamorphose, filtre, passage, monnaie d'échange, palimpseste, voyage, ... les images foisonnent, se superposent et s'effacent, laissant au concept son aura d'irréductibilité. Genette a baptisé quant à lui d'hypertextuelle toute relation qui unit un texte $\mathrm{B}$, l'hypertexte, à un texte antérieur $\mathrm{A}$, l'hypotexte (Genette 1982: 11; 238) ${ }^{2}$. D'un texte l'autre, d'une langue l'autre, la traduction inscrit son processus dans un résultat tangible qui efface ou prétend du moins rendre superflu le texte mère dont il dérive. Alors que dans des transpositions comme la parodie, le pastiche ou la continuation, la réminiscence du texte d'origine fait sens et imprègne l'hypertexte qui en découle, la traduction se veut un produit de substitution qui invite son lecteur à croire qu'il peut se passer de l'hypotexte dont les traces éventuelles sont même la plupart du temps considérées comme perturbatrices, car gênant la bonne compréhension, qui devrait se faire en somme comme si le texte d'origine avait été écrit dans la langue cible. Cet effacement plus ou moins accusé de l'opération traductrice est l'objet d'un enjeu qui varie selon les époques, les sensibilités et les rapports de force culturels et linguistiques. Pascale Casanova a démontré combien l'importation et l'exportation de textes appartenant à des entités linguistiques (et politiques) différentes dessinent un réseau complexe qui révèle en filigrane dans l'espace littéraire d'une époque donnée toute une série de luttes et d'«effets de domination» (Casanova 1999). Regrettant quant à lui que le traducteur fût «méconnu», Larbaud avait plaidé en son temps pour «l'éminente dignité des traducteurs dans la République des Lettres» (Larbaud 1946: 9). Dans toute opération traduisante se manifeste, selon Antoine Berman, «une résistance culturelle» déformatrice dont le traducteur n'est lui-même que l'exécutant plus ou moins conscient: il efface les traces du travail qu'il accomplit en rendant l'Autre conforme au Même, refusant ainsi l' "épreuve de l'étranger» (Berman 1984: 18). L' "invisibilité du traducteur» se voit également dénoncée avec force par Lawrence Venuti, l' «illusion de la transparence» (Venuti 1995: 21, notre traduction) résultant selon lui d'une véritable "violence ethnique de la traduction $»^{3}$ (Venuti 1995: 39, notre traduction). Theo Hermans a démontré pourtant que la voix du traducteur est toujours présente dans la co-émission du discours qu'elle transmet et qu'il y a moyen d'en trouver la trace sans même recourir à la confrontation avec le texte source (Hermans 1996: 42) ${ }^{4}$.

Le transfert d'un ouvrage dans une autre langue, en raison même du changement de perspective et de la prise en compte d'un public nouveau, de normes éditoriales et de traditions culturelles différentes, peut s'accompagner d'un remodelage de l'appareillage paratextuel qui fait remonter à la surface tout l'enjeu traductif. Le péritexte en particulier, dans la mesure où il fournit comme un mode d'emploi contenant des indications sur la manière d'interpréter le texte, peut être soumis à une transformation importante, rendant visible l'opération traductive.

Notre article s'inscrit dans l'axe de toute une série d'études qui ont récemment mis en lumière le rôle majeur joué par la traduction dans les "transferts culturels" (Espagne et Werner 1988). A l'époque des Lumières en particulier, durant tout le «long dix-huitième», l'Angleterre et la France opérèrent des échanges intellectuels qui furent parmi «les plus fructueux de l'histoire européenne» (Rey, Duval et Siouffi 
2007 : 54). Dans l'ouvrage monumental que Gillepsie et Hopkins (2005) ont consacré au phénomène de la traduction en Angleterre, les auteurs démontrent à quel point la frontière entre une œuvre dite originale et sa traduction est tenue et affirment par ailleurs: "it is hard to overstate the importance of translations in the history of anglophone culture» (Gillepsie et Hopkins 2005: viii). Des études comme celles de Hayes (2009), McMurran (2010), Stockhorst (2010) ou encore les contributions parues dans Thomson, Burrows et Dziembowski (2010) appréhendent la traduction comme un enjeu d'intercession essentiel, suscitant des croisements culturels constitutifs à la formation et à l'évolution des cultures dites «nationales». L'ouvrage de McMurran montre ainsi à quel point l'émergence du roman ne saurait être dissociée du champ d'activité de la traduction tant en France qu'en Angleterre; elle insiste en particulier sur l'autonomie et la liberté du traducteur dans ses choix (McMurran 2010: 156). D'une veine analogue, les contributions rassemblées par Stockhorst dans un des rares recueils consacrés à la traduction de la pensée à l'époque des Lumières, décrivent la traduction comme un processus de transfert suscitant acculturation et effervescence créatrice (Stockhorst 2010 : 24). Hayes (2009) a quant à elle déblayé le terrain encore peu exploré de la préface du traducteur entre 1600 et 1800, analysant en particulier la conception que les traducteurs (littéraires) se faisaient de leur travail et la manière dont ils présentaient celui-ci à leurs lecteurs. Elle mentionne qu'il serait tentant d'esquisser les contours d'une typologie des préfaces de traducteurs et établit une liste provisoire d'un certain nombre de fonctions que remplissent les préfaciers qui interviennent pour préciser le contexte historique, pour expliquer ou pour justifier certains aspects de leur travail (Hayes 2009: 8).

Le présent article entend étudier la manière dont la voix principalement herméneutique du traducteur se fait entendre dans un texte de nature philosophique. Nous analyserons pour ce faire un cas précis, celui des éléments péritextuels qui escortent le premier Discours de Jean-Jacques Rousseau dans la version originale et dans les quatre traductions anglaises qui ont paru du vivant du philosophe.

Les témoignages du principal intéressé et de contemporains nous renseignent sur les circonstances de la rédaction et de la publication du premier Discours de Rousseau qui, comme on le sait, est une réponse à un concours organisé par l'Académie de Dijon, dont Rousseau avait découvert le libellé dans un numéro du Mercure de France, alors qu'il se rendait en octobre 1749 à Vincennes réconforter Diderot, enfermé dans le donjon pour avoir publié sa Lettre sur les aveugles (Diderot 1749; voir annexe 1 pour les références aux œuvres). L'ouvrage de Rousseau connut un rare retentissement dont témoignent les périodiques et chroniques du temps ${ }^{5}$. L'éclat fut amplifié encore par une longue polémique faite de réfutations diverses auxquelles Rousseau bien souvent répondit ${ }^{6}$. Alors que l'édition princeps, intitulée Discours qui a remporté le prix à l'Académie de Dijon en l'année 1750 ([Rousseau] 1751a) paraît chez Pissot en janvier 1751 sous une fausse adresse (Barillot \& fils, à Genève) ${ }^{7}$, la première version anglaise paraît à peine six mois plus tard, conjointement à Londres et à Dublin, sans le nom du traducteur ${ }^{8}$. Il s'agit en fait de William Bowyer (16991777), également imprimeur de son état. Son successeur, John Nichols, lui consacra une biographie faite d'anecdotes littéraires, contenant une brève évocation de la découverte de Rousseau en anglais: 
He [William Bowyer] gave likewise to the public, in 1751, with a preface, the first translation that was made of Rousseau's paradoxical oration on the effects of the arts and sciences, which gained the prize at the academy of Dijon, in 1750; and which first announced that singular genius to the attention and admiration of Europe. (Nichols $1782: 222$, voir annexe 1 )

En Angleterre comme sur le continent, Rousseau est d'emblée reçu comme un «auteur à paradoxes ${ }^{9}$, réputation quelque peu simplificatrice dont il aura du mal à se débarrasser, mais qui contribuera à son succès. La version de Bowyer paraît, semble-t-il ${ }^{10}$, quatre fois (Rousseau 1751b; 1751c; 1752a) et est suivie dès 1752 d'une nouvelle traduction, due cette fois à un érudit, le révérend Richard Wynne (1719-1799) $\left(\right.$ Rousseau 1952b) ${ }^{11}$. Huit ans plus tard paraît une troisième traduction de la main d'un auteur qui n'a pu être répertorié (Rousseau 1760, traducteur anonyme [TA]) et en 1767 enfin, l'écrivain satiriste William Kenrick (1725?-1779) édite une série d'ouvrages de Rousseau sous le titre de The Miscellaneous Works of Mr J.J. Rousseau, in five volumes (Kenrick 1767), qui contient, outre des œuvres diverses, parmi lesquelles le Contrat social que Kenrick avait précédemment déjà traduit, une quatrième version anglaise du Discours (Rousseau 1767, traduit par Kenrick) ${ }^{12}$. Les traductions sont toutes les quatre dûment annoncées et font dès leur parution à chaque fois l'objet d'un compte-rendu dans les principaux périodiques de l'époque, tant en Angleterre qu'en Ecosse. Warner (1933, 1940), Sewall (1937) et Roddier (1950) ont en leur temps signalé un large éventail de revues faisant part du premier Discours en Angleterre, comme le Monthly Review (1751: vol 5, 237), le Royal Magazine, or Quarterly Bee (1752: vol. II), le Gentleman's Magazine (1752: vol. XXII et 1753: vol. XXIII), le London Magazine (1760: vol. XXIX), et le Scots Magazine (1768: vol. XXX). Tout en relevant quelques remarques critiques, Sewall est frappé par un accueil globalement positif de la presse et mentionne «the existence of a vigorous, if not widespread, sympathy for Rousseau's ideas during the first twenty years of the reception of his works in England» (Sewall 1937: 911). Les paradoxes que charrient la thèse du citoyen de Genève ont suscité une réception de la même eau, proclamant une sincère admiration pour la tonalité et le fraîcheur du style en dépit des idées exprimées qui sont, elles, reçues avec scepticisme ou dédain ${ }^{13}$. Ainsi, un des tout premiers comptes-rendus parus dans la presse à propos de la traduction de Bowyer louera l'auteur pour son art, mais trouvera à redire sur l'argumentation qui ne serait pas dénuée de sophismes:

[The author is] a compleat master of the declamatory art, which he has powerfully employed on the negative side of the question; but his arguments to shew that the polite arts have ever proved detrimental to plain truth and genuine honesty, have more of sophistry than demonstration in them. (Anonyme Août 1751:237; voir annexe 1)

Ce jugement fait écho à la préface du traducteur dont il reprend le principal argumentaire ainsi qu'il apparaîtra un peu plus loin dans notre analyse. Bien des éléments péritextuels, tant dans la version originale que dans les quatre traductions anglaises, orientent le lecteur dans le déchiffrage d'une polémique qui, chez Rousseau, prend la forme d'«un raisonnement méthodique» mettant en relief, comme le rappelle Michèle Crogiez, "les incohérences et contradictions de l'état de société» (Crogiez 2006: article Paradoxe).

L'édition originale du Discours s'ouvre sur un frontispice en regard de la page de titre. La gravure représente Prométhée apportant le feu à l'homme, qu'un satyre 
semble encourager. La gravure de Charles Bacquoy est faite d'après un dessin de Jean-Baptiste Pierre ${ }^{14}$. "Satyre, tu ne le connais pas» prévient le texte sous l'image, où figure aussi une indication d'une page qui renvoie à l'introït de la deuxième partie du Discours. L'auteur y soutient que les religions anciennes voient dans le dieu qui passe pour l'inventeur des sciences un «ennemi du repos». Rousseau ajoute dans une note en bas de page que son texte évoque la «fable de Prométhée», puis fait allusion à un mythe égyptien analogue avant de conclure: "c'est le sujet du frontispice» (Rousseau 1751/1964: 17) ${ }^{15}$. Les reproches sur les origines viciées des connaissances humaines s'énoncent sous l'égide de Prométhée. Gravure et références à l'histoire de l'Antiquité se déploient comme autant de signes rassurants d'une culture classique maîtrisée ${ }^{16}$ : «L'auteur est très savant et joue par conséquent ici un personnage feint et accommodé à la scène» dira Le Cat au début de sa Réfutation ${ }^{17}$. Aucune des traductions anglaises ne reproduira le frontispice et si la note au début de la seconde partie avec l'allusion à Prométhée est à chaque fois traduite, la phrase «c'est le sujet du frontispice» a quant à elle judicieusement disparu ${ }^{18}$. Dans la Lettre de Jean-Jacques Rousseau, Sur une nouvelle Réfutation de son Discours par un Académicien de Dijon (dite Lettre à Le Cat), Rousseau précisera que le satyre de l'allégorie représente «les hommes vulgaires [...] séduits par l'éclat des Lettres» et que «le Prométhée qui crie et les avertit du danger est le Citoyen de Genève» (Rousseau 1752/1964: 102), qui se voit ainsi investi d'une haute mission véritablement civilisatrice. La gravure contient comme une invite à un processus de transmission et de propagation, y compris dans d'autres langues, car l'art de la gravure est aussi à la peinture ou au dessin ce que la traduction est à la version source; l'une et l'autre se consultent à défaut de l'original ${ }^{19}$. L'absence du frontispice dans les versions anglaises efface du même coup cette allusion à l'opération de transmutation dont elles sont pourtant le résultat et donne à voir comme en abyme le paradoxe d'un Discours qui se construit sur ce qu'il détruit.

\section{La page de titre du Discours et ses traductions}

Retraçant les interventions explicites ou implicites du traducteur, les études que nous avions consacrées à la traduction française de l'Essay concerning human understanding de John Locke (Rooryck et Jooken 2010) ou à celle de la traduction anglaise de L'homme machine de La Mettrie (Jooken et Rooryck 2011) nous avaient permis de dégager ce que nous avions appelé les fonctions argumentative et extra-diégétique assumées par la voix traductive (voir entre autres Rooryck et Jooken 2010: 224). Nous nous proposons d'affiner cette typologie et de distinguer les quatre fonctions suivantes:

1. fonction méta-discursive: le traducteur justifie ses choix ou ses stratégies; il évoque son travail de traducteur;

2. fonction argumentative: le traducteur intervient dans l'argumentation originale pour l'expliciter, la clarifier, la renforcer, voire la contredire;

3. fonction évaluative: le traducteur fait entendre une appréciation positive ou négative à propos du texte qu'il traduit;

4. fonction extra-diégétique: le traducteur incorpore des références contextuelles, tantôt venant de la culture source, tantôt appartenant à la culture cible; il intervient en marge de l'énoncé, en dehors de l'exposé diégétique ${ }^{20}$ proprement dit.

La voix du traducteur, dans la mesure où elle s'approprie la parole étrangère par une interprétation qui prétend lui donner la même valeur que la version originale, 
est assimilable à l'enarratio des anciens traducteurs médiévaux qui revendiquaient l'auctoritas de la Vulgate latine de la Bible pour leurs traductions interprétatives en langage vernaculaire ${ }^{21}$. La traduction se lit ainsi comme un commentaire, tantôt visible, tantôt invisible, accaparant le texte original dans sa métamorphose. La fonction méta-discursive constitue une intervention traductive qui par définition se joue dans le mode de l'explicite, et qui est par conséquent reconnaissable comme telle par le lecteur, car il y est fait allusion à l'art de traduire et à ses difficultés ou ses agréments. Si les fonctions argumentative, extra-diégétique et évaluative peuvent parfaitement être assumées elles aussi par la voix du traducteur, comme dans une préface, une manchette, une note ou un commentaire inséré dans le texte ou dans la marge, elles peuvent tout aussi bien se couler dans le texte, s'intégrer à la parole de l'auteur, ne laissant de trace que si le lecteur a la bonne ou la mauvaise idée de confronter le texte cible avec sa source. Le TA, en ajoutant ainsi au titre «to prove the negative», intervient ostensiblement dans l'argumentation, mais sa parole énarrative n'est visible pour le lecteur du texte cible qu'en regard de l'original.

À l'exception de Wynne, dont le nom figure sur la page de titre $^{22}$, aucune des autres versions anglaises ne donne le nom du traducteur qui demeure ainsi dans l'anonymat, comme souvent au XVIII ${ }^{\mathrm{e}}$ siècle. Le texte, simplement présenté comme traduit («translated from the French» ou «translated from the French original»), fait par là entendre la présence d'une voix discursive nécessairement dédoublée. Dans le cas des Miscellaneous Works, rien n'évoque plus explicitement une traduction. La célébrité de Rousseau étant acquise en 1767, toute référence explicite à l'altérité, qui peut à l'origine séduire un public en quête de nouveauté ou d'étrangeté, se voit ainsi gommée au profit de la désignation d'un auteur appartenant au patrimoine humain. Lauteur du texte était désigné dans les deux premières traductions comme étant un citoyen de Genève ( a citizen of Geneva»), conformément à l'édition originale française $^{23}$. La traduction anonyme de 1760 précise l'identité de l'auteur qu'elle mentionne tant sur le faux titre (A Discourse, by M. Rousseau of Geneva) que sur la page de titre proprement dite. En dix ans, le nom de Rousseau n'est plus inconnu et devient un argument de vente. Les années soixante se voient multiplier les traductions à un rythme qui suit de près les éditions originales ${ }^{24}$ et Kenrick offre en 1767 à ses lecteurs The Miscellaneous Works of Mr. J.J. Rousseau, en omettant cette fois la référence à Genève. Celle-ci avait permis à Rousseau de proclamer une identité qui le distinguait de ses amis encyclopédistes parisiens, tout en faisant entendre une citoyenneté aux accents romains qu'il fera vibrer dans la prosopopée de Fabricius et qui résonnera encore dans le premier livre des Confessions où il évoque ses souvenirs de lecture:

Sans cesse occupé de Rome et d'Athènes; vivant, pour ainsi dire, avec leurs grands hommes, né moi-même Citoyen d'une République, et fils d'un père dont l'amour pour la patrie était la plus forte passion, je m'en enflammois à son exemple; je me croyais Grec ou Romain; [...]. (Rousseau 1782/1959: 9; voir annexe 1)

Pour l'Angleterre anglicane, Genève évoque en outre des valeurs religieuses familières. Henri Roddier rappelait le «sentiment de solidarité à l'intérieur du monde protestant» (Roddier 1950: 16) suite entre autres à la Révocation de l'Edit de Nantes et à la présence de nombreux réfugiés français à Londres ${ }^{25}$.

L'«appareil titulaire» (Genette 1987: 54-97) de l'ouvrage est complexe, tant en raison de sa longueur que parce qu'il contient des informations diverses, qui dépassent la simple fonction de désignation, au point que l'on identifie aujourd'hui 
le plus souvent le texte en parlant plus simplement du Discours sur les Sciences et les Arts ou même du «premier Discours». Le titre d'origine - Discours qui a remporté le prix à l'Académie de Dijon en l'année 1750, sur cette question posée par la même Académie: Si le rétablissement des sciences et des arts a contribué à épurer les mours - est un titre «mixte» comportant à la fois des éléments thématiques (ce livre parle de...) et rhématiques (ce livre est...). Le rhématisme passe ici par une désignation générique: il s'agit d’un discours ${ }^{26}$ en réponse à une question posée par une Académie. Les trois premières traductions parlent bien toutes du prix (praemium ou prize), mais la traduction anonyme laisse tomber la date de 1750 et Kenrick ne fait plus la moindre allusion à la compétition, allant jusqu'à remplacer le terme discourse employé par ses collègues, par le terme dissertation, ôtant du même coup toute référence à la nature initialement oratoire de l'exercice. La séduction (Genette 1987: 87) que le titre doit dégager s'opère ainsi d'abord en renvoyant à un auteur débutant récompensé par un prix, pour ensuite être pleinement assumée dans l'édition de 1767 par le nom de Jean-Jacques Rousseau, auteur connu et reconnu. De même, la restitution de la partie thématique du titre supprime chez Kenrick les allusions au contexte initial. Sa version se contente de déclarer que le texte aborde les effets de la culture des arts et des sciences ("the effects of cultivating the arts and sciences»)" sans renvoi explicite à un impact possible sur les mœurs. Dans les versions antérieures figure la question complète, mais si Bowyer ainsi que le TA optent pour le terme manners en traduction de mours, Wynne écrit morals, se distinguant ainsi de ses collègues ${ }^{28}$. Pour ces derniers la question est de nature sociale: les arts et les sciences épurent-ils (refining et $\underline{\text { rendering pure disent-ils respectivement) }}{ }^{29}$ les usages, les habitudes de vie (manners)? Wynne en revanche a une interprétation qui est plus clairement éthique, tant dans le choix du nom que dans celui du verbe qui l'accompagne, la question étant chez lui de savoir si le rétablissement des arts a contribué à purifier notre morale («the reestablishment of arts and sciences has contributed to purify our morals»). Rappelons en passant que l'Académie de Dijon attribuait bel et bien son prix de Morale ${ }^{30}$. Le traducteur anonyme ajoute quant à lui à la question une indication explicite révélant que le Discours répondra par la négative (proving the negative). Cet ajout argumentatif engage une approche fort dichotomique du débat et accentue ce faisant le caractère polémique du texte, annonçant par la même occasion d'éventuelles répliques épousant un point de vue opposé31.

L'épigraphe Barbarus hic ego sum quia non intelligor illis, tirée des Tristes d'Ovide $^{32}$, figure sous le titre de l'original et des trois premières éditions anglaises; seule la version de Kenrick ignore la citation. Y fait écho un second exergue latin, Decipimur specie recti ${ }^{33}$, sans mention cette fois de l'auteur. Cette référence à l'Art poétique d'Horace (vers 25), que Rousseau avait choisie pour se faire identifier par le jeu d'enveloppes garantissant l'anonymat au concours, est reprise par toutes les éditions anglaises et est placée, comme dans la version originale, après la préface auctoriale, sous la répétition du titre simplifié. Elle précède une sorte de préambule servant d'introduction au texte proprement dit qui commence sous l'intitulé Première partie. Les exergues en latin renforcent l'idée d'appartenance de l'auteur au monde du savoir et des gens cultivés en même temps qu'ils sont une incitation à la réflexion sur la difficulté de saisir une vérité perdue dans le jeu pervers des apparences ${ }^{34}$ : Ovide ne passe pour un sauvage que parce qu'il est loin de Rome, mais les barbares qui l'entourent ne sont pas ceux que le citoyen de Genève côtoie à Paris... 


\section{La voix du traducteur dans les préfaces anglaises}

Avant de passer en revue les différentes fonctions de la Préface, Genette remarque que sa fonction cardinale consiste à "assurer au texte une bonne lecture (Genette 1987: 183). A la voix de l'auteur qui dans une préface oriente la lecture au seuil du texte, peut donc se superposer dans les œuvres traduites celle du traducteur qui, s'il le désire, peut faire entendre explicitement une parole rendant compte d'une manière ou d'une autre de l'altérité linguistique et culturelle du texte qu'il a transféré dans sa langue et veiller lui aussi, compte tenu de ces obstacles supplémentaires, à une bonne lecture, conformément ou non aux visées de l'original. Les trois premières éditions anglaises du Discours contiennent ainsi un texte intitulé The Translator's Preface dans le cas de Bowyer (Bowyer 1751, dans Rousseau 1751a: iii-x) et du traducteur anonyme (Traducteur anonyme 1760, dans Rousseau 1760 : v-vii), alors que la version de Wynne (Wynne 1752, dans Rousseau 1752: vi-viii) se présente comme une sorte d'avertissement s'adressant directement au lecteur: The Translator to the Reader.

L'intervention de Bowyer est nettement plus longue que celle de ses collègues. Elle comprend huit pages contre trois pour le TA et Wynne. Si sa préface est la seule à remplir la fonction argumentative, elle est principalement de teneur évaluative. Bowyer ouvre son texte en motivant son acte de traduction sur une remarque extradiégétique en précisant que le Discours de Rousseau a eu un grand retentissement en France. Il double cette information d'une note évaluative qui qualifie le texte original de singulier: "the following discourse has made such a noise in France, that I thought it, for its singularity, well worth the translating» (Bowyer 1751, dans Rousseau 1751a: iii).

Mais le texte de Rousseau est aussi taxé de dangereux («dangerous»; Bowyer 1751, dans Rousseau 1751a: iii), car l'auteur a pris le mauvais parti dans sa réponse ("he undertook the wrong side of the question»; Bowyer 1751, dans Rousseau 1751a: iv) et Bowyer d'opposer sur le mode de la prétérition un nombre d'arguments en défense des sciences et des arts qui du même coup démontrent que l'auteur, s'opposant à l'opinion établie, a produit un texte qui sortait à ce point de l'ordinaire qu'il méritait d'être traduit et lu. Ce faisant, il renchérit sur la Préface auctoriale originale ${ }^{35}$ : Rousseau prétend en effet d'emblée s'attendre à un "blâme universel» (Rousseau 1751/1964: 3) en raison de l'opinion qu'il défend. Le traducteur est un tiers qui s'interpose entre l'auteur et le lecteur. Dans le discours énarratif qu'est sa Préface, Bowyer estime le Discours réussi malgré son désaccord sur le fond. Détectant chez Rousseau «the art with which he would defeat art» (Bowyer 1751, dans Rousseau 1751a: iv), il met à nu le paradoxe d'un auteur qui maîtrise à merveille l'art oratoire («it must be own'd to be one of the finest modern pieces of oratory», Bowyer 1751, dans Rousseau 1751a: iii) et renonce à combattre l'extravagance de propos analogues aux divagations (raveings) de Hobbes, Agrippa, Spinoza ou Nostradamus (Bowyer 1751, dans Rousseau 1751a: v) ${ }^{36}$ en raison de l'élégance de l'agencement des idées (" yet it must be confessed, his argument is worthy of admiration for its composition and elegance», Bowyer 1751, dans Rousseau 1751a: vii). Evoquant dans sa Préface l'histoire de l'Antiquité pour combattre l'opinion de Rousseau et ayant lui-même recours aux ruses rhétoriques pour appuyer ses arguments, Bowyer reproduit comme en miroir le contrat énonciatif de l'original, mais les voix de l'auteur et de son traducteur ne sauraient entièrement se confondre, car ce que Bowyer légitime dans sa 
Préface n'est pas tant le Discours que la traduction de ce Discours. Aussi termine-t-il son intervention par une remarque méta-discursive sur le mode de l'allusion:

I have endeavoured to keep up to his [ = of Rousseau] spirit in the translation; and if the English reader finds any thing striking in it, I shall put him in mind of the unsuccessful Grecian orator, when he heard his adversary's oration praised on the reading, and, cry out, almost in his words, "What would it be, if you understood the original?" (Bowyer 1751, dans Rousseau 1751a: vii-viii)

En renvoyant à un épisode de l'histoire oratoire grecque, Bowyer fait d'abord allégeance à son auteur, dont il a respecté une pensée qui pourra impressionner le lecteur en raison de la teneur du propos. En même temps il fait comprendre qu'il ne partage pas son avis et qu'il est en fait son contradicteur. Enfin il reconnaît que cet étrange adversaire qu'il sert lui est supérieur. L'allusion historique est d'autant plus significative qu'elle renvoie aux deux plus grands orateurs grecs, Eschine et son rival politique Démosthène. L'anecdote est entre autres reprise par Pline le Jeune dans une Lettre à Népos:

Un jour qu'Eschine lisait à Rhodes la harangue que Démosthène avait faite contre lui, les auditeurs admiratifs applaudissaient. Que serait-ce donc, s'écria-t-il, si vous eussiez entendu le monstre lui-même? (Pline le Jeune $\mathrm{I}^{\mathrm{e}}$ s./1920; voir annexe 1)

Dans les premiers paragraphes de sa Préface, Bowyer avait évoqué dans une amplification les effets terribles que le Discours de Rousseau pourrait avoir s'il était prononcé oralement avec les règles et l'emphase de l'éloquence: «I should tremble for all the libraries of Europe, and dread his elocution almost as much as the fire and swords of Goths, Vandals and Mussulmen» (Bowyer 1751, dans Rousseau 1751a: iii). Ainsi, en filigrane, se substitue à Démosthène, monstre sacré de l'Antiquité37, un nouveau sauvage, un barbare maîtrisant l'art au point d'être capable de le détruire, projet dont Bowyer se désolidarise tout en le colportant. La voix énarrative du traducteur fait entendre comme une réticence qui incorpore une altérité qu'il s'agit malgré tout de reproduire.

Alors que chez Bowyer le nom de Rousseau est mentionné comme une information incidente et peu révélatrice, l'identité de l'auteur devient dans les préfaces des traductions suivantes un élément qui gagne sans cesse en importance. Dans un premier temps Wynne refuse de prendre position dans le débat. Il évoque (métadiscursivement) son travail de traducteur en soulignant sa fidélité au texte: «I [...] shall present the reader with the author's sentiment, without deviating from them in the least» (Wynne 1752 dans Rousseau 1752b: vii-viii). Il précise qu'en France le Discours a été à la fois applaudi et condamné et affirme d'emblée: «I shall not presume to say any thing on either side of the question» (Wynne 1752 dans Rousseau 1752: vii). S'il évite par conséquent toute remarque argumentative, sa parole se fait dans un deuxième temps solidaire de celle de l'auteur, comme en témoigne la présence de la préface auctoriale qui précède ici l'intervention du traducteur et rend plus perméable la frontière séparant l'original de sa traduction. Wynne accumule les remarques extra-diégétiques concernant les polémiques que le Discours a suscitées en France et se montre très au fait des réactions publiées entre autres dans le Mercure de France. Il mentionne ainsi successivement la réponse du roi Stanislas de Pologne, la réplique de l'auteur, la réaction de «one M. Gautier» (Wynne 1752 dans Rousseau 1752: vii) et la Lettre de Rousseau à Grimm, précisant la date et la signature: «in 
November 1751, and signed with his name I.I. Rousseau» (Wynne 1752 dans Rousseau 1752: vii). Si le rôle évaluatif du traducteur se limite à laisser le lecteur juge et arbitre, Wynne ne peut s'empêcher de se moquer de Gautier, Rousseau ayant selon lui démontré dans sa lettre à Grimm «the weakness and absurdity of M. Gautier's arguments» (Wynne 1752 dans Rousseau 1752: vii). Pour lui, l'Académie a récompensé un discours «superior in merit» et il évoque une traduction possible des réactions de Rousseau à ses contradicteurs, du moins si le public se montre curieux, "as well on the account of the subject it treats of, as on that of its author" (Wynne 1752 dans Rousseau 1752: vii). Derrière le texte se profile bientôt la personne de Rousseau, qui devient, par le truchement d'anecdotes biographiques, un argument de vente.

Le traducteur anonyme (TA) présente en 1760 une préface fort proche de celle de Wynne. Il prétend ne pas vouloir se mêler au débat et n'entre donc pas dans l'argumentation: "whether his conclusions are rightly drawn, we pretend not to determine» (TA, dans Rousseau 1760:v). Il renchérit sur les informations extra-diégétiques en mentionnant à son tour explicitement les textes du roi Stanislas et de Gautier ainsi que les réponses de Rousseau, tout en écornant au passage la réponse du roi de Pologne qu'il qualifie en une remarque évaluative de « very superficial and unworthe the attention of our author» (TA, dans Rousseau 1760 : vi). Le reste du texte ne tarit pas d'éloges sur l'auteur. Celui qui est identifié sur la page de titre comme $M$. Rousseau of Geneva vivrait conformément aux principes qu'il défend dans ses ouvrages. Sa doctrine est brièvement évoquée en termes virulents. Se rendre utile est un devoir de citoyen: "He is of opinion that an useless membre of society is in some degree criminal» (TA, dans Rousseau 1760: vi). Jouir du superflu relève d'un goût $\mathrm{du}$ luxe fort condamnable car "what a man enjoys more than is neccessary for the Support of Life, is Luxury, consequently culpable». Rousseau gagne sa vie en copiant de la musique «for which he charges a very low price». Il fuit l'abondance et se limite au nécessaire, comme en témoigne une anecdote sur une somme d'argent qu'il refuse parce qu'elle représenterait davantage que son travail de copiste ne méritait. Ces informations extra-diégétiques corroborent les nouvelles qui circulaient dans les journaux de l'époque; elles contribuent à la création d'un personnage dont l'extravagance et le comportement peu ordinaire constituaient un atout que les libraires n'hésitaient pas à exploiter pour stimuler leurs ventes, laissant à Rousseau son goût de la sobriété et de la réserve.

\section{De l'art de la déviation}

Venuti plaidait pour des traductions reconnaissant «the linguistic and cultural difference of foreign texts » (Venuti 1995: 41). Nous nous sommes limités dans la présente contribution à examiner des éléments péritextuels du premier Discours de Rousseau sans entrer plus avant dans le texte, mais il n'y a pas lieu de parler ici d'un phénomène outrancier d'acculturation. L'étrangeté du Discours sur les Sciences et les Arts frappe en France comme en Angleterre par une singularité qui remet en question un certain optimisme de progrès tel qu'il se dessine dans la société occidentale à l'époque des Lumières et qu'il se proclame avec force dans l'Encyclopédie qui paraît au même moment ${ }^{38}$. La "présentation de soi» du locuteur (la voix du citoyen de Genève, bientôt de Rousseau de Genève, relayée ou non par celle d'un traducteur à 
travers la voix énarrative) est ici comme en porte-à-faux avec l'argumentation défendue dans le Discours qu'il énonce. La prise de parole se fait selon les poncifs d'usage, avec un appareil titulaire conforme aux coutumes éditoriales, précédant un Discours qui inscrit le déroulement de son argumentation dans la tradition oratoire et rhétorique, sans rechigner sur les citations et les références. Or c'est précisément pour dénoncer ces sciences et ces arts, au sein desquels il occupe désormais une place de marque par le prix qui le récompense, que l'orateur prend la parole. Rousseau déploie avec le génie qui lui est propre l'art des lettres qu'il s'évertue à vouer aux gémonies.

Un détournement analogue se produit à son égard dans les traductions anglaises. La voix du narrateur original se dédouble et est prise en relais par des préfacierstraducteurs qui se mettent au service d'une parole étrangère dont ils s'approprient. Cette appropriation de nature herméneutique revendique l'autorité de la version originale mais laisse des traces où se fait entendre le traducteur dont la voix énarrative assume les fonctions méta-discursive, argumentative, évaluative et extra-diégétique. Dans leur teneur principalement évaluative, les préfaces énarratives des traducteurs brossent l'image d'un écrivain extrêmement doué qui prend un parti contraire au bon sens. Les premières préfaces sont clairement évaluatives et argumentatives, alors que l'anonyme et Kenrick incorporent dans leur évaluation des anecdotes extra-diégétiques célébrant la réputation de Rousseau.

Tous les péritextes vont ce faisant très précisément à l'encontre des intentions de l'auteur: "On ne demande plus d'un livre s'il est utile, mais s'il est bien écrit» déplore Rousseau dans les derniers paragraphes de son Discours (Rousseau 1751/1964: 25) Son texte se voit pourtant apprécié pour sa prouesse rhétorique et est ainsi récupéré par le système même qu'il dénonce. La même chose se produit à propos d'anecdotes biographiques qui servent de faire-valoir à l'œuvre: Rousseau, devenu célèbre, est applaudi pour son refus de l'ostentation et du luxe, et ce refus même est affiché sans retenue afin d'attirer les lecteurs. En 1761, l'Emile est brûlé à Paris et condamné à Genève. L'ouvrage interdit paraît prestement dans deux traductions anglaises simultanées, l’une proposée par Kenrick (1762), l'autre par Nugent (1763). Nugent regrette dans sa Préface que certains enferment son auteur dans «a paradoxical way of thinking, of which he has been accused in his other performances» (Nugent 1763 dans Rousseau 1763:x), mais il ne l'en présente pas moins comme «the ingenious Rousseau, an author who has already merited the public applause by many ingenious performances» (Nugent 1763 dans Rousseau 1763: viii).

S'emparant de Rousseau comme dans un ultime dévoiement énarratif, les traducteurs anglais n'hésiteront pas à exploiter les idées paradoxales du citoyen de Genève en y voyant bientôt l'expression individuelle d'une liberté de pensée que la France ou Genève restreignent à coups de censure et que l'Angleterre seule est en mesure de propager:

It is perhaps in England alone that our author can hope to see his book fairly submitted to the judgement and candour of the public. (Kenrick 1762 dans Rousseau 1762: I, iv)

La voix énarrative du traducteur fait entendre que le texte qu'elle interprète bénéficie, dans sa forme nouvelle et indispensable, du public qu'il mérite et s'approprie ainsi en filigrane le statut de l'autorité de l'original. 


\section{NOTES}

1. Sur les métaphores de la traduction, voir entre autres la mise au point de Hewson (2006).

2. «La forme de transposition la plus voyante, et à coup sûr la plus répandue, consiste à transposer un texte d'une langue à une autre: c'est évidemment la traduction, [...]» (Genette 1982: 11,238)

3. Venuti parle respectivement d'illusion de transparence («illusion of transparency»; Venuti 1995: 21) et de violence ethnocentrique de la traduction («ethnocentric violence of translating»; Venuti 1995: 39).

4. «[...] the Translator's voice is always present as co-producer of the discourse» (Hermans 1996: 42). Alors que Hermans avait étudié un texte de fiction, notre propos porte sur un discours philosophique et prendra également en compte les marques que seule rend visibles une confrontation avec l'original.

5. Voir en particulier le chapitre que Raymond Trousson (2000: 11-57) consacre à la première publication de Rousseau.

6. Dès 1753, l'éditeur Jean-Paul Mevius rassembla pas moins de 19 textes autour du Discours (Mevius 1753a; 1753b, voir annexe 1).

7. Pissot est un libraire parisien. Sur les raisons de ce subterfuge, voir entre autres l'article Pissot dans le Dictionnaire Rousseau (Trousson et Eigeldinger: 2006) et le catalogue de l'exposition sur Rousseau et les arts visuels (Collectif 2001: 39).

8. Les ouvrages classiques de Joseph Texte (1895) et de Henri Roddier (1950) demeurent riches en informations sur les échanges entre la France et l'Angleterre à l'époque de Rousseau. Roddier mentionne les quatre traductions du Discours sur les Sciences et les Arts (1950: 30-33).

9. On songe à la Réponse du roi Stanislas à propos du Discours: «N'est-ce qu'un paradoxe dont il [l'auteur] a voulu amuser le public?» (Mevius 1753a: 62, voir annexe 1).

10. L'édition de 1752 (Rousseau 1752a, traduit par Bowyer) mentionne "the fourth edition" sans que nous ayons trouvé trace de la troisième.

11. Wynne a publié des ouvrages religieux, des essais sur le langage et une traduction du nouveau testament en regard de la version grecque. Voir l'article paru lors du décès de Wynne dans The European Magazine and London Review (Anonyme 1799, voir annexe 1).

12. Le premier volume des Miscellaneous Works (1767) comprend outre le premier Discours, la réponse du roi Stanislas, la lettre de Rousseau à Grimm, la «réponse finale» de Rousseau et le Discours sur l'inégalité.

13. Duffy résume la double attitude de la presse en ces termes: «the typical English response to him was $[\ldots]$ a comfortable simultaneity of disregard for Rousseau's judgements and admiration for his writings» (Duffy 1979: 16).

14. On retrouvera une reproduction et une description du frontispice dans Jean-Jacques Rousseau face aux arts visuels (Hurley 2001 : 91-92).

15. Nous renvoyons aux textes de Rousseau dans l'édition en cinq volumes des Euvres complètes de la Bibliothèque de la Pléiade $(1959 ; 1961 ; 1964 ; 1969 ; 1995)$. Le Discours sur les Sciences et les Arts figure dans le volume 3 (Rousseau 1751/1964).

16. Rousseau cite Plutarque et Platon en raccourci afin de mieux étayer son argumentation. Voir entre autres Trousson (1964/2001: 277, note 84) et Masters (2002: 271-272).

17. Voir Mevius (1753b: 11, annexe 1). La Réfutation de Le Cat avait paru sans nom précédée du Discours «du citoyen de Genève» sous l'adresse «à Londres, chez Edouard Kelmarneck, 1751». Cette édition comporte le frontispice.

18. Phrase supprimée elle aussi dans les éditions françaises anciennes (entre autres dans les contrefaçons) qui n'ont pas le frontispice. Voir les descriptions de Dufour (1925: I, 15-18).

19. Voici ce que dit Claude-Henri Watelet à l'article Gravure de L’Encyclopédie (tome VII, page 888): «Le graveur est pour les peintres dont il imite les tableaux, ce que le traducteur est pour les auteurs dont il interprete les ouvrages; ils doivent l'un et l'autre conserver le caractere de l'original, et se dépouiller de celui qu'ils ont; ils doivent être des protées: on ne lit une traduction, et l'on ne consulte pour l'ordinaire une gravure, que pour connoître les auteurs originaux». Voir aussi Lavezzi (2003).

20. Par diégèse, nous entendons l'univers de l'œuvre originale (qui ici est de nature argumentative) sur laquelle vient se greffer la voix énarrative.

21. Sur le sens que les Anciens donnaient au terme enarratio et la dimension herméneutique de la traduction des textes sacrés, voir Damian-Grint (1999).

22. Voir, dans l'annexe 2, un aperçu des informations contenues dans les pages de titre des quatre versions anglaises. 
23. Sur les trois éditions qui paraissent chez Pissot en 1751 figure "par un citoyen de Genève»; la même année paraît une quatrième édition à Genève, chez Barillot, avec cette fois la mention «par M. Rousseau de Genève» (Trousson 2000: 12). Bowyer dévoilait déjà le nom de l'auteur dans sa préface: "All I can learn of the author is, that his name is Rousseau; and that he is, as he says, of Geneva, or of some place in the neighbourhood of Switzerland» (Bowyer 1751, dans Rousseau 1751c: iii). D’un point de vue biographique l'on sait que Rousseau, en raison de sa conversion au catholicisme, avait en réalité perdu sa citoyenneté genevoise qu'il allait recouvrer en 1754 .

24. Eloisa, or, a Series of original Letters, 1761; Emilius and Sophia: or, a new system of Education, 1762; A Treatise on the Social compact, or the Principles of Political Law, 1764. Ces trois œuvres majeures ont été traduites par Kenrick de façon anonyme. Le Contrat social est repris dans le volume 4 des Miscellaneous Works.

25. Warner (1933: 476-477, note 21) insiste sur la récurrence du nom de Rousseau associé à celui de Genève, «a protestant state», ajoute-t-il «with which England was particularly friendly, undoubtedly tended to increase his popularity in the latter country».

26. L'auteur s'inscrit ainsi dans toute une tradition, elle-même ancrée dans ce que Marc Fumaroli a appelé L'âge de l'éloquence (1980). L'abbé Mallet, qui rédige l'article Discours pour l'Encyclopédie (1754: IV, 1033-1034), explique que ce genre oratoire consiste à assembler "des raisonnements réunis et disposés suivant le style de l'art»; les Discours s'adressent «directement à l'esprit» et doivent «expliquer et instruire», mais ils peuvent aussi «toucher» et «n'excluent pas toute passion».

27. Toutes les version anglaises parlent de Arts and Sciences, alors que dans le Discours original figure l'ordre inverse, qui est aussi celui de L'Encyclopédie (ou Dictionnaire raisonné des Sciences, des Arts et des Métiers). Choix impliquant quelque préséance épistémologique ou simple affaire d'euphonie? «La spéculation et la pratique constituent la principale différence qui distingue les Sciences d'avec les Arts", écrit d Alembert dans son Discours préliminaire de l'Encyclopédie. Et d'ajouter : «Il faut pourtant bien avouer que nos idées ne sont pas encore bien fixées sur ce sujet» (D'Alembert 1751/1986: 106).

28. Dans sa préface Bowyer utilise toutefois les deux termes - $\underline{\text { morals }}$ et manners - comme des notions solidaires: "This gentleman would have us believe that the arts and sciences have injured our morals and manners» (Rousseau 1751c: vi). Gourevitch signale dans sa traduction moderne la difficulté que représente la traduction du mot mours pour lequel n'existe pas de réel équivalent anglais: "Mours is notoriously difficult to translate. No single English word consistently means 'shared public morality'. 'Manners' might seem possible, but the burden of Rousseau's numerous discussions of mœurs is the discrepancy between manners and morals.» (Gourevitch 1997, dans Rousseau XVIII s./1997: xlv).

29. Le critique du The Monthly Review (Août 1751: 237) juge faible le titre de Bowyer et n'hésite pas à suggérer une alternative qui explicite l'aspect dichotomique de la question: "The question is ill expressed in the title-page, as above. It might have been translated thus. 'Whether the re-establishment of arts and sciences, has contributed to improve or corrupt our manners?'».

30. Voir le texte de l'annonce dans le Mercure de France d'octobre 1749 dans Rousseau (1751b/1964: 1237).

31. Une traduction anglaise de la réponse faite par le roi Stanislas ([Stanislas, Roi de Pologne] 1752; voir annexe 1) accompagnée des Observations de Rousseau faites à cette réponse paraîtront chez R. James (l'éditeur de Bowyer) dès 1752. Le nom de Gautier y figure dans une orthographe fantaisiste (Guatier) au lieu de celui du roi. Kenrick propose une traduction différente dans les Miscellaneous. Notons aussi deux répliques originales anglaises parues sans nom d'auteur à Dublin en 1751, Education display'd et A reply to the Discourse which carried the promium at the Academy of Dijon (cette dernière réplique est parfois attribuée à Samuel Madden).

32. «Ici c'est moi le barbare, puisqu'ils ne me comprennent pas». La citation figure aussi en exergue aux (voir Rousseau 1782/1959: 657) et traverse ainsi toute l'œuvre de Rousseau.

33. «Nous sommes trompés par l'apparence du bien».

34. Les ouvrages de Jean Starobinski (comme Jean-Jacques Rousseau - La transparence et l'obstacle, 1971) ont dégagé comme on sait toute l'importance que prend la thématique de l'être et du paraître chez Rousseau.

StARobinski, Jean (1971): Jean-Jacques Rousseau - La transparence et l'obstacle. Paris: Gallimard.

35. La Préface de Rousseau est reprise dans les quatre traductions: The Authors's Preface (Rousseau 1751c: ix-x); The Preface of the Author (qui précède ici l'avertissement du traducteur, Rousseau 1752 : iii-v); The Preface (Rousseau 1760: vii-viii); Preface (Rousseau 1767: 1-2). 
36. Rousseau cite lui-même dans son Discours «les dangereuses rêveries de Hobbes et de Spinoza" (Rousseau 1751/ 1964: 28). Bowyer juge apparemment leurs systèmes philosophiques aussi excentriques que les ouvrages ésotériques de Nostradamus et de l'occultiste Agrippa de Nettesheim (1486-1535).

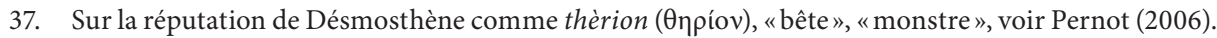

38. Dans son Discours préliminaire de l'Encyclopédie, d'Alembert (1751/1986; voir annexe 1) évoque explicitement le Discours de Rousseau, qui avait collaboré à l'entreprise par ses articles concernant la musique. Il applaudit à l'éloquence tout en se distanciant des propos tenus.

\section{RÉFÉRENCES}

Berman, Antoine (1984): L'épreuve de l'étranger. Paris: Gallimard.

Casanova, Pascale (1999): La République mondiale des Lettres. Paris: Seuil.

Crogiez, Michèle (2006): Paradoxe. In: Trousson, Raymond et Eigeldinger, Frédéric S. Dictionnaire de Jean-Jacques Rousseau. Paris: Honoré Champion.

Damian-Grint, Peter (1999): Translation as Enarratio and Hermeneutic Theory in TwelfthCentury Vernacular Learned Literature. Neophilologus. 83(3):349-367.

Duffy, Edward (1979): Rousseau in England: the Context for Shelley's Critique of the Enlightenment. Berkeley: University of California Press.

Dufour, Théophile (1925): Recherches bibliographiques sur les cuvres imprimées de J.-J. Rousseau. Vol. 1. Paris: L. Giraud-Badin.

ESPAGNE, Michel et Werner, Michael, dir. (1988): Transferts: les relations interculturelles dans l'espace franco-allemand (XVIII ${ }^{e}$ et XIX ${ }^{e}$ siècles). Paris: Éditions Recherches sur les Civilisations.

Fumaroli, Marc (1980): L'âge de l'éloquence. Genève: Droz.

Genette, Gérard (1982): Palimpsestes. Paris: Seuil.

Genette, Gérard (1987): Seuils. Paris: Seuil.

Gillespie, Stuart et Hopkins, David, dir. (2005): The Oxford History of Literary Translation in English. Volume 3: 1660-1790. Oxford: Oxford University Press.

Hayes, Julie Candler (2009): Translation, Subjectivity, and Culture in France and England, 1600-1800. Stanford: Stanford University Press.

Hermans, theo (1996): The Translator's Voice in Translated Narrative. Target. 8(1):23-48.

Hewson, Lance (2006): Evolution et emprise des métaphores de la traduction. In: Michel BALlard, dir. Qu'est-ce que la traductologie? Arras: Artois Presses Université, 271-281.

Hurley, Cecilia, dir. (2001): Jean-Jacques Rousseau face aux arts visuels. Neuchâtel: Bibliothèque publique et universitaire de Neuchâtel.

Jooken, Lieve et Rooryck, Guy (2011): The Freedom of Expressing One's Ideas: Translating La Mettrie. The Translator. 17(2):233-254.

Larbaud, Valéry (1946): Sous l'invocation de Saint Jérôme. Paris: Gallimard.

Lavezzi, Elisabeth (2003) : Rousseau et les discours sur la gravure. Annales de la société JeanJacques Rousseau. 45:313-334.

MAsters, Roger D. (2002): La philosophie politique de Rousseau. Lyon: ENS Editions.

McMurran, Mary Helen (2010): The Spread of Novels. Translation and Prose Fiction in the Eighteenth Century. Princeton/Oxford: Princeton University Press.

Pernot, Laurent (2006): L'Ombre du Tigre. Recherches sur la réception de Démosthène. Naples: M. D'Auria Editore.

Rey, Alain, Duval, Frédéric et Siouffi, Gilles (2007): Mille ans de langue française: histoire d'une passion. Tome II: Nouveaux destins. Paris: Perrin.

Roddier, Henri (1950): J.-J. Rousseau en Angleterre au XVIII siècle. L'œeuvre et l'homme. Paris: Boivin.

Rooryck, Guy et Jooken, Lieve (2010): Locke ou la traduction de l'entendement. In: Ton NaAijkens, dir. Event or Incident. On the Role of Translations in the Dynamics of Cultural Exchange. Bern: Peter Lang, 211-246.

Sewall, Richard B. (1937): Rousseau's First Discourse in England. Publications of the Modern Language Association of America. 52(3):908-911. 
Starobinski, Jean (1971): Jean-Jacques Rousseau: la transparence et l'obstacle. Paris: Gallimard. SтоскноRsт, Stefanie, dir. (2010): Cultural Transfer through Translation. Amsterdam/New York: Rodopi.

Texte, Joseph (1895): Jean-Jacques Rousseau et les origines du cosmopolitisme littéraire: étude sur les relations littéraires de la France et de l'Angleterre au XVIII siècle. Paris: Hachette.

Thomson, Ann, Burrows, Simon et Dziembowski, Edmond, dir. (2010): Cultural Transfers: France and Britain in the Long Eighteenth Century. Oxford: Voltaire Foundation.

Trousson, Raymond (2000): Jean-Jacques Rousseau jugé par ses contemporains. Du «Discours sur les sciences et les arts» aux «Confessions». Paris: Honoré Champion.

Trousson, Raymond (1964/2001): Le thème de Prométhée dans la littérature européenne. Genève: Droz.

Trousson, Raymond et Eigeldinger, Frédéric S. (2006): Dictionnaire de Jean-Jacques Rousseau. Paris: Honoré Champion.

Venuti, Lawrence (1995): The Translator's Invisibility. London/New York: Routledge.

WARner, James H. (1933): The Reaction in Eighteenth-Century England to Rousseau's Two Discours. Publications of the Modern Language Association of America. 48(2):471-487.

WARner, James H. (1940): The Basis of J.-J. Rousseau's Contemporaneous Reputation in England. Modern Language Notes. 55(4):270-280.

\section{ANNEXE 1}

\section{Sources primaires}

Les œuvres dont les noms d'auteurs sont entre crochets ont été publiés anonymement, mais leur auteur a été identifié.

Anonyme (Août 1751): Monthly Catalogue. The Monthly Review. 5:237.

Anonyme (1751a): Education display'd. Being an answer to the oration which carry'd the promium at the Academy of Dijon, in 1750, on this question proposed by the academy 'Whether the re-establishment of arts and sciences has contributed to the refining of manners?' By a merchant of Dublin. Dublin: Printed by Augustus Long.

Anonyme (1751b): A reply to the Discourse which carried the praemium at the Academy of Dijon, in 1750. On this question, proposed by the said Academy, hath the re-establishment of arts and sciences contributed to purge or corrupt our manners? In a letter to the author. Dublin: printed for Peter Wilson [attribué parfois à Samuel Madden].

Anonyme (Août 1799): Monthly Obituary. The European Magazine and London Review. 36:70.

D'Alembert, Jean le Rond (1751/1986): Discours préliminaire de l'Encyclopédie. In: Denis Diderot et Jean d'Alembert Encyclopédie, ou dictionnaire raisonné des sciences, des arts et des métiers. Paris: Garnier-Flammarion.

Diderot, Denis (1749): Lettre sur les aveugles à l'usage de ceux qui voient. Londres, [Paris, Durand].

Diderot, Denis et d'Alembert, Jean le Rond (1751-1772/2011) Encyclopédie ou dictionnaire raisonné des sciences, des arts et des métiers. In: Robert MORISSEY, dir. ARTFL Encyclopédie Project (édition du printemps 2011). Chicago: University of Chicago. Consultée le 29 mars 2012, <http://encyclopedie.uchicago.edu $>$.

Kenrick, William, dir. (1767): The Miscellaneous Works of Mr. J.J. Rousseau, in five volumes. London: T. Becket \& P.A. De Hondt.

Masters, Roger et Kelly, Christopher, dir. (1997): Collected Writings of Rousseau. Volume 2. Discourse on the Sciences and Arts (First Discourse) and Polemics. (Traduit par ) Hanover, Dartmouth College: University Press of New England.

Mevius, Jean-Paul, dir. (1753a): Recueil de toutes les pièces qui ont été publiées à l'occasion du Discours de M. J.J. Rousseau sur cette question proposée par l'Académie de Dijon pour le Prix de l'année 1750. Si le rétablissement des sciences \& des arts a contribué à épurer les mours. Vol. 1. Gotha: Chez Jean-Paul Mevius. 
Mevius, Jean-Paul, dir. (1753b): Recueil de toutes les pièces qui ont été publiées à l'occasion du Discours de M. J.J. Rousseau sur cette question proposée par l'Académie de Dijon pour le Prix de l'année 1750. Si le rétablissement des sciences \& des arts a contribué à épurer les mours. Vol. 2. Gotha: Chez Jean-Paul Mevius.

Nichols, John (1782): Biographical and Literary Anecdotes of William Bowyer, printer, FSA. London: Printed by and for the Author.

Pline Le Jeune ( $\mathrm{I}^{\mathrm{e}}$ s./1920): Pline à Nepos. Lettres. Livre second, III. (Traduit par Louis Sylvestre DE SACY et Jules Amable Pierrot) Paris: Garnier Frères éditeurs Consulté le 12 Décembre 2013, <http://www.roma-quadrata.com/plinelettres.html\#Livre2>.

[Rousseau, Jean-Jacques] (1751a): Discours qui a remporté le prix à l'Académie de Dijon en l'année 1750. Sur cette question posée par la même Académie: Si le rétablissement des Sciences et des Arts a contribué à épurer les mours. Par un citoyen de Genève: Barillot \& fils.

Rousseau, Jean-Jacques (1751b): The Discourse which Carried the Praemium at the Academy of Dijon [...]. By a Citizen of Geneva. (Traduit par William Bowyer) London: Owen.

Rousseau, Jean-Jacques (1751c): The Discourse which Carried the Praemium at the Academy of Dijon [...]. By a Citizen of Geneva. (Traduit par William Bowyer) Dublin: James.

Rousseau, Jean-Jacques (1751/1964): Discours sur les Sciences et les Arts. In: Jean-Jacques Rousseau Euvres complètes. Vol. 3. Paris: Gallimard, 1-30; notes 1237-1256.

Rousseau, Jean-Jacques (1752a): The Discourse which Carried the Praemium at the Academy of Dijon [...]. By a Citizen of Geneva. (Traduit par William Bowyer) Dublin: Faulkner et James.

Rousseau, Jean-Jacques (1752b): A Discourse, to which the Prize was adjudged by the Academy of Dijon [...]. By a Citizen of Geneva. (Traduit par Richard Wynne) London: Withers.

Rousseau, Jean-Jacques (1752/1964): Lettre de Jean-Jacques Rousseau - Sur une nouvelle Réfutation de son Discours par un Académicien de Dijon. In: Jean-Jacques RousseAu. Euvres complètes. Vol. 3. Paris: Gallimard, 97-102.

Rousseau, Jean-Jacques (1760): A Discourse by M. Rousseau of Geneva. [...] (Traducteur anonyme) London: Burnet.

Rousseau, Jean-Jacques (1762): Emilius and Sophia: or, a New System of Education. 4 vol. (Traduit par William KenRICK) London: R. Griffiths, T. Becket and P.A. De Hondt.

Rousseau, Jean-Jacques (1763): Emilius: Or, an Essay on Education. 2 vol. (Traduit par Thomas Nugent, 1763) London: J. Nourse and P. Vaillant.

RousseAu, Jean-Jacques (1767): A Dissertation on the effects of cultivating the arts and sciences. In: Kenrick, William, dir. The Miscellaneous Works of Mr. J.J. Rousseau, in five volumes (Traduit par William Kenrick) London: T. Becket \& P.A. De Hondt, I, 1-43.

Rousseau, Jean-Jacques 1782/1959): Les Confessions. Livre 1. In: CEuvres complètes. Vol. 1. Paris: Gallimard, 1-44.

RousseAu, Jean-Jacques (1782/1959): Les Dialogues. Rousseau juge de Jean-Jacques 1. In: CEuvres complètes. Vol. 1. Paris: Gallimard, 657 - 989.

RousseAu, Jean-Jacques (xviII ${ }^{e}$ s./1959) : Euvres complètes. Vol. 1. Paris: Gallimard.

Rousseau, Jean-Jacques (XVIII $\left.{ }^{\mathrm{e}} \mathrm{s} . / 1961\right)$ : Euvres complètes. Vol. 2. Paris: Gallimard.

Rousseau, Jean-Jacques (xviII s./1964): CEuvres complètes. Vol. 3. Paris: Gallimard.

Rousseau, Jean-Jacques (XviII ${ }^{\mathrm{e}}$ s./1969): Euvres complètes. Vol. 4. Paris: Gallimard.

Rousseau, Jean-Jacques (xviII s./1995): CEuvres complètes. Vol. 5. Paris: Gallimard.

Rousseau, Jean-Jacques (xviII ${ }^{\mathrm{e}}$ s./1997): The Discourses and Other Early Political Writings. (Traduit par Victor Gourevitch) Cambridge: Cambridge University Press.

[Stanislas, Roi de Pologne] ([1752]): An Answer to the Discourse which carried the Promium at the Academy of Dijon; On the question, Whether the re-establishment of arts and sciences hath contributed to the refinement of manners? By Monsieur Guatier [sic], Professor of Mathematicks and of History, and Fellow of the Royal Academy of Belles-Lettres, at Nancy. To which are added, Observations on the above Answer to that Discourse. By John-James Rousseau, Citizen of Geneva, Author of the Discourse. (Traducteur anonyme) Dublin: Richard James. 
ANNEXE 2

Pages de titre

Édition originale

\section{DISCOURS \\ QUI A REMPOR'TE! J.F PRIX}

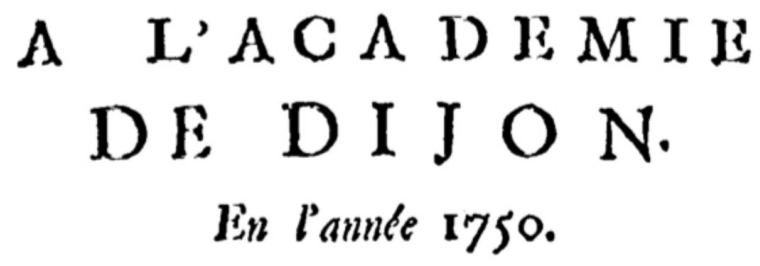

Sur cette Queftion propofée par la même Académie :

Si le rétablifjement des Sciences $\mathcal{E}$ des Arts a contribué dépurer les maurs.

par un Citoyen de Gentev.

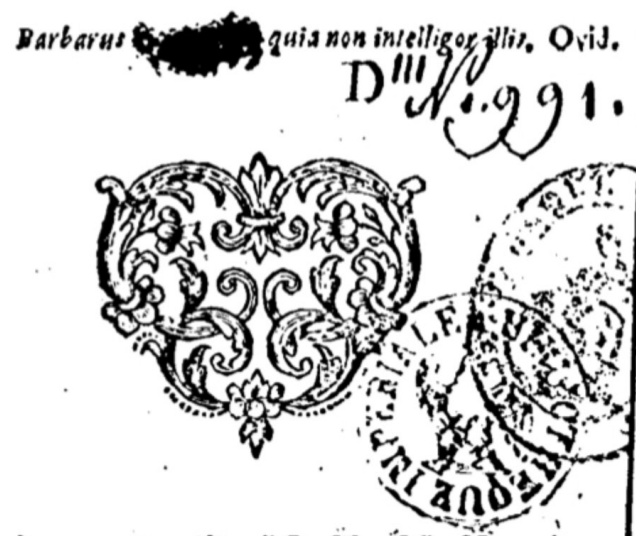

A G E N E V E;

Chez BAR IL L OT \& fils,

Libre de droit, Jean-Jacques Rousseau [domaine public], via Wikimedia Commons. Consulté le 15 décembre 2013, <http://commons.wikimedia.org/wiki/File \%3ARousseauDiscourseSciencesArt.jpg>. La citation d'Ovide se lit comme suit: «Barbarus hic ego sum, qui a non intelligor illis». 


\begin{tabular}{|c|c|c|c|}
\hline \multicolumn{4}{|c|}{ Traductions anglaises } \\
\hline Bowyer 1751 & Wynne 1752 & Anonyme 1760 & Kenrick 1767 \\
\hline $\begin{array}{c}\text { THE } \\
\text { DISCOURSE } \\
\text { Which Carried the } \\
\text { PRAEMIUM } \\
\text { AT } \\
\text { The ACADEMY of DIJON, } \\
\text { in MDCCL. } \\
\text { On this QUESTION, } \\
\text { Propos'd by the said } \\
\text { Academy, Whether the } \\
\text { Re-establishment of ARTS } \\
\text { and ScIENCEs has } \\
\text { contributed to the } \\
\text { refining of Manners. } \\
\text { By a Citizen of GENEVA. } \\
\text { Barbarus hic ego sum, } \\
\text { quia non intelligor illis. } \\
\text { Ovid. } \\
\text { Translated from the } \\
\text { FRENCH Original. } \\
\text { DUBLIN: } \\
\text { Printed by RICHARD } \\
\text { JAMES, at Newton's Head, } \\
\text { in Dame-Street, 1751. } \\
\text { LONDON, } \\
\text { Printed for W. OwEN, } \\
\text { near Temple-Bar. } \\
\text { MDCCLI. } \\
\text { [Price One Shilling.] }\end{array}$ & $\begin{array}{c}\text { By a Citizen of Geneva. } \\
\text { Barbarus hic ego sum, } \\
\text { quia non intelligor illis. } \\
\text { Ovid. } \\
\text { Translated from the } \\
\text { French, by R. WynNe, } \\
\text { A.M. } \\
\text { LONDON } \\
\text { Printed for E. Withers, } \\
\text { at the Seven Stars, } \\
\text { opposite Chancery-Lane, } \\
\text { in Fleet Street, 1752. } \\
\text { [Price One Shilling.] }\end{array}$ & $\begin{array}{l}\text { D I S C O U R S E } \\
\text { B Y } \\
\text { M. ROUSSEAU of Geneva, } \\
\text { Which got the Premium at } \\
\text { the Academy of Dijon, on } \\
\text { this Question proposed by } \\
\text { the said Academy, Whether } \\
\text { the Revival of the Arts and } \\
\text { Sciences has contributed to } \\
\text { render our Manners pure? } \\
\text { proving the Negative. } \\
\text { Barbarus hic ego sum, quia } \\
\text { non intelligor illis. Ovid. } \\
\text { Translated from the } \\
\text { FRENCH. } \\
\text { [ornement typographique] } \\
\text { L O N D O N: } \\
\text { Printed for G. BuRNET, } \\
\text { near Temple-Bar. } \\
\text { MDCCLX. }\end{array}$ & $\begin{array}{c}\text { THE } \\
\text { MISCELLANEOUS } \\
\text { W O R K S } \\
\text { OF } \\
\text { Mr. J.J. ROUSSEAU. } \\
\text { IN FIVE VOLUMES. } \\
\text { VOLUME I. } \\
\text { [ornement } \\
\text { typographique] } \\
\text { LONDON: } \\
\text { Printed for T. BECKET } \\
\text { and P.A. DE HoNDT, in } \\
\text { the Strand. } \\
\text { MDCCLXVII. } \\
\text { (Page de titre) } \\
\text { A } \\
\text { DISSERTATION } \\
\text { ON THE } \\
\text { E F F E C T S } \\
\text { OF CULTIVATING THE } \\
\text { ARTS AND SCIENCES. } \\
\text { Decipimur Specie recti. } \\
\text { (p.3) }\end{array}$ \\
\hline
\end{tabular}

